\title{
Neumonía: La pandemia ignorada
}

\author{
Pneumonia: The forgotten pandemic \\ Neumonía: La pandemia ignorada
}

\section{Enrique Gea-Izquierdo}

enriquegea@yahoo.es

https://orcid.org/0000-0002-7123-6251

Pontificia Universidad Católica del Ecuador, Facultad de Medicina, Quito, Ecuador

Recibido 18 de junio 2021 / Arbitrado y aceptado 29 de junio 2021 / Publicado 13 de diciembre 2021

Palabras clave: Infecciones respiratorias;

Neumonía; Epidemiología

\section{Infecciones respiratorias y neumonía}

En algunos países la infección aguda del tracto respiratorio inferior causa más enfermedad y muerte que cualquier otra infección; como es el caso de Estados Unidos. De hecho, estas infecciones generan también una mayor carga de enfermedad en todo el mundo que la infección por el virus de la inmunodeficiencia humana (VIH), la malaria, el cáncer o los ataques cardíacos (1). En lo relativo a la neumonía la prevalencia de varios patógenos y la epidemiología de la enfermedad varían ampliamente entre países y regiones, lo que dificulta la discusión precisa de la carga internacional de la enfermedad.

\section{Epidemiología de la neumonía}

Anualmente, la neumonía afecta aproximadamente a 450 millones de personas, el siete por ciento del total mundial, y provoca unos 4 millones de muertes. Tiene presencia mundial, pero la infección es más frecuente en las áreas subdesarrolladas, especialmente en partes del sur de Asia y África subsahariana. En países en desarrollo se ha estimado una incidencia mundial de casos de neumonía clínica de 0,29 eventos por niño y año, lo cual implica 150,7 millones de episodios nuevos al año, mientras que en el mundo desarrollado no se dispone de datos comparables. La incidencia estimada de neumonías clínicas presenta su mayor valor en el sureste de Asia (0,36 eventos por niño y año), por delante de África $(0,33)$ y el Mediterráneo oriental $(0,28)$, y requieren 
hospitalización por neumonía grave de 7-13 $\%$ de los casos. En el mundo desarrollado la incidencia anual de neumonía se estima en torno a $33 \times 10.000$ en niños menores de 5 años y $14,5 \times 10.000$ en niños de 0 a 16 años. Sin embargo, la incidencia de la NAC en niños menores de 5 años es aproximadamente 0,026 episodios por niño y año, lo que induce que más del $95 \%$ de todos los casos mundiales de neumonía clínica en niños pequeños acontecen en los países en desarrollo (2,3). La neumonía aguda en los Estados Unidos es la sexta causa más común de muerte y la primera en infección relacionada con la mortalidad. La incidencia es de 170-280 / 10.000, los costos de tratamiento superan los 12 billones de dólares y el tratamiento hospitalario cuesta 25 veces más que el tratamiento ambulatorio. En 2003, la tasa de mortalidad ajustada por edad causada por influenza y neumonía fue de 20,3 por 100.000 personas (4). Las estimaciones de la incidencia de NAC varían de 4 millones a 5 millones de casos por año, y alrededor del 25 $\%$ requieren hospitalización (5). Se estima que $\mathrm{NAH}$ se produce en 250.000 personas por año, lo que representa alrededor del 15 al $18 \%$ de todas las infecciones nosocomiales $(6,7)$.

Otros autores consideran que la carga y la epidemiología de la enfermedad han sido difíciles de describir con suficiente precisión debido a la falta de una definición adecuada de neumonía con fines epidemiológicos y de pruebas sensibles y específicas para establecer la etiología bacteriana (8). A pesar de importantes avances, las dimensiones del problema clínico planteado por la neumonía siguen siendo enormes. Después de los logros iniciales alcanzados con los antibióticos de primera generación, la tasa de mortalidad por neumonía no ha cambiado de manera apreciable en los últimos 50 años (9). En general, la mortalidad es del $1 \%$ en pacientes no hospitalizados, $13,7 \%$ en pacientes hospitalizados, $19,6 \%$ en pacientes con bacteriemia y $<36,5 \%$ en unidades de cuidados intensivos.

Por relevancia, la epidemiología de las infecciones bacterianas por neumonía incluye: Streptococcus pneumoniae, Haemophilus influenzae tipo B, Staphylococcus aureus, Escherichia coli, Pseudomonas aeruginosa y Salmonella no tifoidea. Para el caso de Streptococcus pneumoniae representa $>50$ $\%$ de la bacteriemia. Indicar, que la incidencia de Streptococcus pneumoniae y Haemophilus influenzae podría ser menor en las regiones donde se administran las vacunas; sin embargo, existe un mayor riesgo de Haemophilus influenzae $B$, enfermedad meningocócica invasiva.

\section{Epidemiología de la neumonía en niños}

Es conocido que la neumonía es una enfermedad respiratoria que puede resultar mortal (10). Especialmente afecta a niños en países en desarrollo (11), presentándose cada año más de 150 millones de casos de neumonía, requiriendo hospitalización en más de una decena de millón (2). Neumonía y diarrea son consideradas las dos mayores causas de muerte en el mundo en niños 
menores de cinco años $(12,13)$. Sin embargo, es posible la reducción de la mortalidad asociada con la neumonía en la comunidad. De hecho, la OMS apunta que la adopción de políticas adecuadas y la promoción de la salud son fundamentales para este fin (14). Asimismo, la asignación de recursos económicos dirigidos a programas específicos y la integración de actividades para el tratamiento de la neumonía a nivel comunitario son aspectos básicos para la disminución de la mortalidad. Adicionalmente, la OMS indica que el fomento de prácticas de prevención y tratamiento, junto con la implicación de las entidades gubernamentales, no gubernamentales y comunitarias, mejoraría la asistencia sanitaria de primer nivel; reforzando los lazos entre los centros sanitarios, su personal y la comunidad.

La neumonía es la principal causa individual de mortalidad infantil en todo el mundo, superando en niños menores de cinco años al total de muertes por el sida, malaria y sarampión (15). Diversos autores han descrito esta mortalidad en el mundo $(12,16$ 18) a pesar de la dificultad para realizar las estimaciones y las diferencias entre el mundo desarrollado y no desarrollado, calculando que en la actualidad hay más de 2 millones de muertes cada año en niños menores de 5 años (19). Sin embargo, la distribución de la mortalidad para la enfermedad es desigual (20), sobresaliendo África con un $45 \%$ de las muertes totales atribuibles para esta enfermedad (21) contrastando con una baja tasa de mortalidad en países desarrollados (22). Por el contrario, las tasas de mortalidad en niños en la mayoría de los países en desarrollo varían entre 60 a 100 por 1.000 nacidos vivos y una quinta parte de estas muertes se debe a la neumonía (15). Por lo tanto, la neumonía provoca aproximadamente un $19 \%$ de todas las muertes acontecidas en el mundo en niños menores de cinco años $(3,12)$, estando fuertemente vinculada a la malnutrición, falta de acceso a cuidados sanitarios y pobreza (23).

La enfermedad constituye la principal causa de muerte infantil en niños por debajo de 5 años en países con desarrollo medio o bajo. En este grupo de edad la enfermedad registra una mortalidad mundial anual del $18 \%$, que corresponde un $20 \%$ a países poco desarrollados y un $4,3 \%$ a desarrollados (13). En el año 2000 se estimaron 13,8 millones de casos de neumonía neumocócica con 741.000 muertes en niños menores de 5 años, la mayoría de ellos acontecidos en el sureste de Asia (38,6\%), África $(27,6 \%)$ y la región del Pacífico occidental (17\%) (24). Respecto a Haemophilus influenzae se estima unos 7,9 millones de casos mundiales anuales de neumonía para la misma edad, lo que supone 292.000 muertes (25) aunque la incidencia de neumonía en niños disminuye con la edad (2). Estimaciones más recientes (11) muestran que para el 2011, 1,3 millones de neumonías fueron mortales, con una alta proporción de muertes en los dos primeros años de vida.

Hay que resaltar que tres cuartas partes de las neumonías presentes en niños menores de cinco años acontecen en 15 países, destacando India y China, y estos representan $74 \%$ de los casos anuales mundiales (3). Tal y como se ha 
indicado, para el descenso de la incidencia de la enfermedad es necesario la realización de intervenciones de carácter preventivo, en especial: la vacunación periódica, alimentación complementaria, lactancia exclusiva, reducción de la contaminación del aire interior (26) y administración de suplementos de zinc $(27,28)$. Además, el aumento en la calidad de la atención sanitaria y la asistencia en la comunidad ayuda a reducir eficazmente la enfermedad (29). De hecho, los programas comunitarios (30) para el tratamiento de la neumonía han indicado que el uso correcto de antibióticos es una medida eficaz para combatirla y la vacunación contra la tosferina, el sarampión y el Haemophilus influenzae tipo b contribuirían a disminuir la incidencia de las infecciones de las vías respiratorias.

En el 2007 la neumonía representaba casi el $20 \%$ de las muertes en el mundo (31), seguida de la diarrea (13). Tal y como se ha indicado a nivel mundial fallecen todos los años aproximadamente 1,8 millones de niños menores de cinco años, con representación de más de un $98 \%$ de las muertes por neumonía en 68 países (32); principalmente en Asia Sudoriental y África, por lo que puede considerarse una pandemia. Además, se estima que entre 11 y 20 millones de niños con neumonía al año requieren hospitalización y que más de 2 millones morirán como consecuencia de la enfermedad(15).

\section{REFERENCIAS BIBLIOGRÁFICAS}

1. Mizgerd JP. Acute lower respiratory tract infection. N Engl J Med. 2008;358(7):716-27.
2. Rudan I, Tomaskovic L, Boschi-Pinto C, Campbell $\mathrm{H}$. The Child Health Epidemiology Reference Group. Global estimates of the incidence of clinical pneumonia among children under five years of age. Bull World Health Organ. 2004;82(12):895-903.

3. Rudan I, Boschi-Pinto C, Biloglav Z, Mulholland K, Campbell H. Epidemiology and etiology of childhood pneumonia. Bull World Health Organ. 2008;86(5):408-16.

4. Hoyert DL, Heron MP, Murphy SL, Kung HC. Deaths: Final data for 2003. National vital statistics reports; Hyattsville, MD: National Center for Health Statistics. 2006; 54(13)

5. American Thoracic Society. Guidelines for the initial management of adults with communityacquired pneumonia: diagnosis, assessment of severity, and initial antimicrobial therapy. Am J Respir Crit Care Med. 2001;163:1730-54.

6. Wiblin RT, Wenzel RP. Hospital-acquired pneumonia. Curr Clin Top Infect Dis. 1996;16:194-214.

7. Bassin AS, Niederman MS. New approaches to prevention and treatment of nosocomial pneumonia. Semin Thorac Cardiovasc Surg. 1995;7:70-7.

8. Cherian T. Describing the epidemiology and aetiology of bacterial pneumonia in children: an unresolved problem. JHPN. 2005;23(l):1-5.

9. Wunderink RG, Mutlu GM. Pneumonia: overview and epidemiology. In: Laurent GJ, Shapiro SD, eds. Encyclopedia of Respiratory Medicine. Oxford, UK: Elsevier Academic Press. 2006.

10. Gea-Izquierdo E. Impacto de la neumonía en España: 1992-2001. Rev Cubana Hig Epidemiol. 2014;52(3):346-56.

11. Walker $C L$, Rudan I, Liu L, Nair $H$, Theodoratou E, Bhutta ZA, et al. Global burden of childhood pneumonia and diarrhoea. Lancet. 2013;381(9875):1405-16. 
12. Bryce J, Boschi-Pinto C, Shibuya K, Black RE; WHO Child Health and Epidemiology Research Group. WHO estimates of the causes of death in children. Lancet. 2005;365:1147-52.

13. Black RE, Cousens $S$, Johnson HL, Lawn JE, Rudan I, Bassani DG, et al. Child Health Epidemiology Reference Group of WHO; UNICEF. Global, regional and national causes of child mortality in 2008: A systematic analysis. Lancet. 2010;375(9730):1969-87.

14. Organización Mundial de la Salud/UNICEF. Declaración conjunta de la OMS y el UNICEF. Tratamiento de la neumonía en el medio comunitario. 2004.

15. The United Nations Children's Fund. WHO (2006). Pneumonia: The forgotten killer of children. New York: UNICEF [citado 21 dic 2017]. Disponible en: http://www.childinfo.org/ files/Pneumonia_The_Forgotten_Killer_of_ Children.pdf

16. Garenne $\mathrm{M}$, Ronsmans $\mathrm{C}$, Campbell $\mathrm{H}$. The magnitude of mortality from acute respiratory infections in children under 5 years in developing countries. World Health Stat Q. 1992;45:180-91.

17. World Bank. World development report 1993: Investing in health. Washington, DC: Oxford University Press. 1993.

18. Williams BG, Gouws E, Boschi-Pinto C, Bryce $J$, Dye C. Estimates of world-wide distribution of child deaths from acute respiratory infections. Lancet Infect Dis. 2002;2:25-32.

19. Morris SS, Black RE, Tomaskovic L. Predicting the distribution of under-five deaths by cause in countries without adequate vital registration systems. Int J Epidemiol. 2003;32(6):1041-51.

20. United Nations (2017). United Nations Millennium Development Goals. [citado 21 dic 2017]. Disponible en: http://www.un.org/apps/ news/story.asp?NewsID=44639
21.WHO (2017). World Health statistics. Geneva: WHO; 2007 [citado 21 dic 2017]. Disponible en: http://www.who.int/whosis/whostat2007.pdf

22. Jokinen $\mathrm{C}$, Heiskanen $\mathrm{L}$, Juvonen $\mathrm{H}$, Kallinen S, Karkola K, Korppi M, et al. Incidence of community-acquired pneumonia in the population of four municipalities in eastern Finland. Am J Epidemiol. 1993;137(9):977-88.

23. WHO/UNICEF. Global action plan for prevention and control of pneumonia (GAPP). Geneva: WHO. 2009.

24. O'Brien KL, Wolfson LJ, Watt JP, Henkle E, Deloria-Knoll M, McCall N, et al. Burden of disease caused by Streptococcus pneumoniae in children younger than 5 years: Global estimates. Lancet. 2009;374(9693):893902.

25. Watt JP, Wolfson $\sqcup, O^{\prime}$ Brien $K L$, Henkle $E$, Deloria-Knoll M, McCall $N$, et al. Burden of disease caused by Haemophilus influenzae type $b$ in children younger than 5 years: Global estimates. Lancet. 2009;374(9693):903-11.

26. Dherani M, Pope D, Mascarenhas M, Smith $K R$, Weber $M$, Bruce N. Indoor air pollution from unprocessed solid fuel use and pneumonia risk in children aged under five years: Systematic review and metaanalysis. Bull World Health Organ. 2008;86:390-8.

27. Black RE. Zinc deficiency, infectious disease and mortality in the developing world. J Nutr. 2003;133(5):1485S-9S.

28. Brooks WA, Santosham $M$, Naheed $A$, Goswami D, Wahed MA, Diener-West $M$, et al. Effect of weekly zinc supplements on incidence of pneumonia and diarrhoea in children younger than 2 years in an urban, lowincome population in Bangladesh: Randomised controlled trial. Lancet. 2005;366(9490):9991004. 
29. WHO. Departament of Child and Adolescent Health and Development Family and Community Health. Management of pneumonia in community settings. Ginebra: UNICEF-WHO. 2004.

30. Dawson $P$, Pradhan $Y V$, Houston R, Karki $S$, Poudel D, Hodgins S. From research to national expansion: 20 years' experience of community-based management of childhood pneumonia in Nepal. Bull World Health Organ. 2008;86:339-43.
31. WHO. The Global Burden of Disease: 2004 update. Geneva: WHO. 2008.

32. The United Nations Children's Fund. Tracking progress in maternal, neonatal and child survival: The 2008 report. Genebra: UNICEF-WHO. 2008.

Conflicto de intereses: Ninguno declarado por el autor. Financiación: Ninguna declarada por el autor.

Agradecimiento: Ninguno manifestado por el autor.

\section{ACERCA DEL AUTOR}

Enrique Gea-Izquierdo. PhD, MPH, MOH \& S, MSc. Doctor, Mención Doctor Internacional. Máster en Investigación y Avances en Medicina Preventiva y Salud Pública. Máster en Prevención de Riesgos Profesionales. Máster en Gestión de Riesgos Laborales, Calidad y Medio Ambiente. Licenciado en Ciencias Biológicas. Diplomado en Profesorado de Educación. Pontificia Universidad Católica del Ecuador, Ecuador. 